Vietnam Journal of Mechanics, VAST, Vol.29, No. 1 (2007), pp. 25-36

\title{
SPECTRAL PROPER TRANSFORMATION AND APPLICATION TO GUST RESPONSE PREDICTION OF STRUCTURES
}

\author{
LE THAi HoA \\ Wind Engineering Laboratory, \\ Graduate School of Engineering, Kyoto University, Japan \\ NGUYEN DONG ANH \\ Institute of Mechanics, VAST
}

\begin{abstract}
Random turbulent loading on engineering structures which immersed in the atmospheric turbulent flow is often represented as the multi-dimensional and/or multivariate Gaussian random loading processes. Gust response prediction, however, usually burdens a lot of computational difficulties due to turbulent loading projection on the generalized structural coordinates. In these cases, the decomposition techniques must be required to decouple the multi-variate turbulent loading into the independently generalized turbulent forces, then is associated with the generalized structural modes. This paper will present the proper orthogonal decomposition using the spectral proper transformation in the frequency domain to decouple the multi-variate turbulent loading processes. New approach in the gust response prediction of structures will be formulated with numerical example of cable-stayed bridge.
\end{abstract}

\section{INTRODUCTION}

Gust response prediction of structures subjected to the turbulent-induced forces in the atmospheric turbulent flow requires as a must among wind êffects and wind-induced vibrations. General formulation of gust response prediction of line-like structures has proposed by Davenport [6]. As a principle, the multi-degree-of-freedom (MDOF) motion equations of structures were decomposed orthogonally in the generalized coordinates and vibrational mode shapes thanks to the structural modal transformation (SMT). However, there were inevitable difficulties to generalize external turbulent forces, which then are associated with generalized coordinates. So far, the joint acceptance function (JAF) has been applied to determine the generalized turbulent forces and gust response analysis of bridges in both the frequency and time domains $[4,6]$.

Proper orthogonal decomposition (POD), known as Karhunen-Loeve expansion has been applied for many engineering fields such as discrete signal processing, random field decomposition and turbulence analysis, data and image compression, system identification and control, probabilistic dynamic response and so on $[2,13,14]$. In the wind engineering, the POD has been used in analysis of random pressure field $[1,17]$, bluff-body flow identification and related physical phenomena [7], random field decomposition and stochastic dynamic response [3,12]. Basing upon a formulation of basic matrix in the proper transformation, the POD has been divided into two branches: $i$. the covariance-matrixbased POD and covariance proper transformation $(\mathrm{CPT})$ in the time domain and ii. the 
spectral-matrix-based POD and spectral proper transformation (SPT) in frequency domain $[3,5,16]$. In the former, the basic matrix is based on the zero-time-lag covariance matrix of turbulent loading processes in the time domain, whereas cross spectral density matrix of these processes used in the frequency domain in the later. It is generally agreed that almost literatures, however, have been discussed on the covariance-based POD and CPT in the time domain. The spectral-based POD and SPT is very promising to apply for stochastic response analysis, but it burdens much in complicated computation ratter than the CPT. Recently, new approach of the gust response analysis of structures, so-called double modal transformations (DMT) has been proposed by Carassale et al. [3], by which the structural modes decomposed by the SMT are associated with the so-called orthogonally turbulent loading modes decomposed by the SPT to determine the gust response in both the generalized and structural coordinates. Advantage of the POD applications on the gust response analysis of structures has hinged on comprehensive approach for the generalized gust force formulation in which the fully-correlated turbulent field is directed accounted. Especially, the lowest turbulent loading modes that have been decomposed from the fullycorrelated turbulent field thanks to the POD analysis can contribute dominantly on the structurally generalized responses of the lowest structural modes. The DMT using the the spectral-based POD and SPT has been applied for the gust response prediction of simple frames, buildings by some authors $[3,5,16]$, and for that of bridges $[9,18]$. Time domain gust response analysis of bridges using the covariance-based POD and CPT has firstly presented by Le and Nguyen [10,11]. In previous applications of spectral-based DMT, however, the simple quasi-steady theory has been accounted for the turbulent-induced forces.

In this paper, the spectral-matrix-based POD and its SPT will be presented and application to decoupling the multi-variate turbulent loading processes. New comprehensive approach on the gust response prediction of structures then will be formulated using the SPT with emphasis on numerical example of cable-stayed bridge. The turbulent-induced forces based on corrected quasi-steady theory with aerodynamic admittance also are used for more refinement.

\section{SPECTRAL PROPER TRANSFORMATION}

The main idea of the POD is to find out a set of orthogonal vector basis which can represent a multi-variate random process into a sum of products of these basic orthogonal vectors and single-variant uncorrelated random processes. The spectral-based orthogonal vectors are found as the eigenvector solutions of the eigen problem of the cross spectral density matrix as:

$$
S_{v}(n) \Psi_{v}(n)=\Lambda_{v}(n) \Psi_{v}(n),
$$

where $S_{v}(n)$ : cross spectral density matrix formed from multi-variate random process $v(t) ; \Lambda_{v}(n), \Psi_{v}(n)$ : spectral eigenvalue and eigenvector matrices $\Lambda_{v}(n)=\operatorname{diag}\left(\lambda_{v 1}(n)\right.$, $\left.\lambda_{v 2}(n), \ldots \lambda_{v N}(n)\right), \Psi_{v}(n)=\left[\psi_{v 1}(n), \psi_{v 2}(n), \ldots \psi_{v N}(n)\right] ; n$ : frequency variable; $\psi_{v j}(n)$ : spectral eigenvector associated with spectral eigenvalue $\lambda_{v i}(n)$.

Since the cross spectral density matrix $S_{v}(n)$ is a Hermitian and positive definite one, thus its spectral eigenvalues are real and positive, its spectral eigenvectors are generally complex, satisfy the orthonormal conditions as follows:

$$
\Psi_{v}^{* T}(n) \Psi_{v}(n)=I ; \Psi_{v}^{* T}(n) S_{v}(n) \Psi_{v}(n)=\Lambda_{v}(n) .
$$


Thus, the Fourier transform and the cross spectral density matrix of $v(t)$ can be represented in terms of the orthogonal eigenvectors $\Psi_{v}(n)$ as follows:

$$
\begin{gathered}
\hat{v}(n)=\Psi_{v}(n) \hat{y}_{v}(n)=\sum_{j=1}^{N} \psi_{v j}(n) \hat{y}_{v j}(n), \\
S_{v}(n)=\Psi_{v}(n) \Lambda_{v}(n) \Psi_{v}^{* T}(n)=\sum_{j=1}^{N} \psi_{v j}(n) \lambda_{v j}(n) \psi_{v j}^{* T}(n),
\end{gathered}
$$

where $\hat{y}_{\nu}(n)$ : turbulent principal coordinates as Fourier transforms of uncorrelated singlevariate random processes; $N$ : dimension of cross spectral matrix $S_{v}(n) ; *, T$; denote to complex conjugate and transpose operation.

The spectral-based quantities can be approximately reconstructed by using limited number of the lowest spectral eigenvectors (assumed that the spectral eigenvectors rearranged in reduced order of their corresponding spectral eigenvalues) as follows:

$$
\begin{aligned}
& \hat{v}(n)=\sum_{j=1}^{\hat{M}} \psi_{v j}(n) \hat{y}_{v j}(n) \\
& S_{v}(n)=\sum_{j=1}^{\hat{M}} \psi_{v j}(n) \lambda_{v j}(n) \psi_{v j}^{* T}(n),
\end{aligned}
$$

where $\hat{M}$ : number of truncated spectral eigenvectors $(\hat{M}<N)$. This approximation is known as the spectral proper transformation (SPT) in the frequency domain.

\section{SPATIALLY-CORRELATED TURBULENT FIELD AND TURBULENT-INDUCED FORCES}

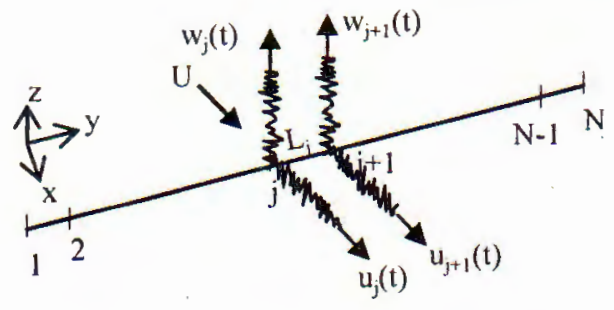

Fig.1. Turbulent loading processes

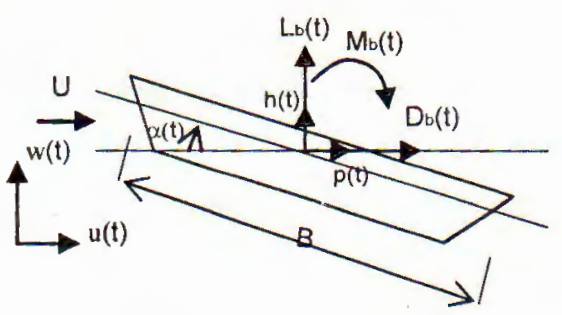

Fig. 2. Sectional tubulent forces

Considering a line-like structure horizontally immersed in an atmospheric turbulent flow, the turbulent-induced loading that discretized at the structure's nodes are mainly induced by longitudinally and vertically turbulent fluctuations $u(t), w(t)$ (laterally turbulent component $v(t)$ is omitted due to very small effect). These turbulent components, furthermore, are considered as uncorrelated with each other. Thus, the random turbulent field acting on the structure's $N$ - nodes can be represented as two $N$ - variate zero-mean Gaussian random processes, see Fig. 1:

$$
u(t)=\left\{u_{1}(t), u_{2}(t), \ldots, u_{N}(t)\right\}^{T} ; \quad w(t)=\left\{w_{1}(t), w_{2}(t), \ldots, w_{N}(t)\right\}^{T} .
$$


Spatially-correlated $N$-variate random turbulent field, in which elements at two separated points are spatially coherent together can be represented by symmetrically-squared cross spectral matrix:

$$
\begin{aligned}
S_{v} & =\left[\begin{array}{llll}
S_{v_{1} v_{1}}(n) & S_{v_{1} v_{2}}(n) & \ldots & S_{v_{1} v_{N}}(n) \\
S_{v_{2} v_{1}}(n) & S_{v_{2} v_{2}}(n) & \ldots & S_{v_{2} v_{N}}(n) \\
\ldots & \ldots & \ldots & \ldots \\
S_{v_{N} v_{1}}(n) & S_{v_{N} v_{2}} & \ldots & S_{v_{N} v_{N}}(n)
\end{array}\right] \\
& =\left[S_{v_{m} v_{k}}(n)\right]_{(N \times N)} ; \quad
\end{aligned}
$$

where $v$ denotes $u(t)$ or $w(t) ; S_{v}$ : cross spectral matrices; $S_{v_{m} v_{k}}(n)$ : cross power spectral elements between $v_{m}(t)$ and $v_{k}(t)$ at nodes $m, k ; N$ : number of structure's nodes. Cross spectral elements are determined from available auto spectral densities as:

$$
S_{v_{m} v_{k}}(n)=\sqrt{S_{v_{m} v_{m}}(n) S_{v_{k} v_{k}}(n)} \operatorname{coh}_{v}\left(n, \Delta_{m k}\right),
$$

where $S_{v_{m} v_{m}}(n), S_{v_{k} v_{k}}(n)$ : auto spectral densities of $v(t)$ at nodes $m, k$, respectively; $\operatorname{coh}_{v}\left(n, \Delta_{m k}\right)$ : coherence function between two spectral components at separated nodes $m, k$ along structure's axis which can be obtained due to the available empirical model such as. $[6,15]$ :

$$
\operatorname{coh}_{v}\left(n, \Delta_{m k}\right)=\exp \left(-\frac{c_{v} n\left|y_{m}-y_{k}\right|}{0.5\left(U_{m}+U_{k}\right)}\right)
$$

where $U_{m}, U_{k}$ : mean velocities at nodes $m, k ; c_{v}$ : decay factor; $\Delta_{m k}=\left|y_{m}-y_{k}\right|$ : distance between nodes $m$ and $k ; y_{m}, y_{k}$ : longitudinal coordinates of $m, k$ along structure's axis.

Uniform turbulent-induced forces per unit structural length (consisting of lift, drag, moment: $L_{b}(t), D_{b}(t), M_{b}(t)$, see Fig. 2 are determined from random turbulent field $u(t), w(t)$ due to the corrected quasi-steady theory [6], in which frequency-dependant aerodynamic admittance functions are supplemented.

$$
\begin{gathered}
L_{b}(t)=\frac{1}{2} \rho U^{2} B\left[C_{L}\left(\alpha_{0}\right) \chi_{L u}(n) \frac{2 u(t)}{U}+\left(C_{L}^{\prime}\left(\alpha_{0}\right)+C_{D}\left(\alpha_{0}\right)\right) \chi_{L w}(n) \frac{w(t)}{U}\right] \\
D_{b}(t)=\frac{1}{2} \rho U^{2} B\left[C_{D}\left(\alpha_{0}\right) \chi_{D u}(n) \frac{2 u(t)}{U}+\left(C_{D}^{\prime}\left(\alpha_{0}\right)-C_{L}\left(\alpha_{0}\right)\right) \chi_{D w}(n) \frac{w(t)}{U}\right] \\
M_{b}(t)=\frac{1}{2} \rho U^{2} B^{2}\left[C_{M}\left(\alpha_{0}\right) \chi_{M u}(n) \frac{2 u(t)}{U}+C_{M}^{\prime}\left(\alpha_{0}\right) \chi_{M w}(n) \frac{w(t)}{U}\right]
\end{gathered}
$$

where $C_{L}, C_{D}, C_{M}$ : aerodynamic static coefficients at balanced angle of attack $\alpha_{0}$ (usually $\left.\alpha_{0}=0^{\circ}\right) ; C_{L}^{\prime}, C_{D}^{\prime}, C_{M}^{\prime}$ : first derivatives with respect to angle of attack at balanced angle $C_{F}^{\prime}=\left.\frac{d C_{F}(\alpha)}{d \alpha}\right|_{\alpha_{0}=0}, F=L, D, M ; \chi_{F v}(n)(F=L, D, M ; v=u, w)$ : aerodynamic transfer functions between turbulent components and turbulent-induced forces (their absolute magnitudes refer as aerodynamic admittance functions); $\rho, B, U$ : air density, width and mean velocity, respectively.

Then full-scale turbulent-induced forces acting on whole structure can be formulated:

$$
F_{b}(t)=\frac{1}{2} \rho U B\left[C_{u} \chi_{u F}(n) u(t)+C_{w} \chi_{w F}(n) w(t)\right],
$$




$$
\begin{array}{ll}
F_{b}(t)=\left[\begin{array}{l}
L_{b i}(t) \\
D_{b i}(t) \\
M_{b i}(t)
\end{array}\right] ; & C_{u}=\frac{1}{2} \rho U B\left[\begin{array}{l}
2 C_{L} \bar{L} \\
2 C_{D} \bar{L} \\
2 B C_{M} \bar{L}
\end{array}\right] ; \quad C_{w}=\left[\begin{array}{l}
C_{L}^{\prime} \bar{L} \\
C_{D}^{\prime} \bar{L} \\
B C_{M}^{\prime} \bar{L}
\end{array}\right] ; \\
\bar{L}=\operatorname{diag}\left(L_{i}\right) ; & L_{i}=\left\{\begin{array}{l}
0.5\left|y_{2}-y_{1}\right|, \quad i=1 \\
0.5\left|y_{i+1}-y_{i-1}\right|, 1<i<N \\
0.5\left|y_{N}-y_{N-1}\right|, \quad i=N
\end{array}\right.
\end{array}
$$

where $C_{u}, C_{w}$ : full-scale force coefficient matrices; $y$ : longitudinal structural coordinate.

\section{SPECTRAL-BASED GUST RESPONSE FORMULATION}

The MDOF motion equation of structure in the atmospheric turbulent flow subjected to the turbulent-induced forces is expressed:

$$
M \ddot{U}+C \dot{U}+K U=F_{b}(t),
$$

where $M, C, K$ : globally mass, damping and stiffness matrices, respectively; $U, \dot{U}, \ddot{U}$ : deflection vector and its derivative vectors; $F_{b}(\mathrm{t})$ : full-scale turbulent-induced forces.

Decomposing into the mass matrix-based normalized generalized coordinates and the structural mode shapes using the $\operatorname{SMT}\left(\Phi^{T} M \Phi=I ; \Phi^{T} K \Phi=\Omega ; U=\Phi \xi=\sum_{i=1}^{\bar{M}} \phi_{i} \xi_{i}\right)$, thus IDOF motion equation in the $i^{\text {th }}$ generalized coordinate excited by generalized turbulentinduced forces is obtained:

$$
\ddot{\xi}_{i}+2 \zeta_{i} \omega_{i} \dot{\xi}_{i}+\omega_{i}^{2} \xi_{i}=\phi_{i}^{T} F_{b}(t),
$$

where $\Phi$ : modal matrix; $\Phi=\left[\phi_{1}, \phi_{2}, \ldots, \phi_{M}\right] ; \phi_{i}: i^{\text {th }}$ mode; $\xi_{i}: i^{\text {th }}$ generalized coordinates; $\omega_{i}, \zeta_{i}$ : circular frequency and damping ratio; $\bar{M}$ : number of truncated structural modes $(\bar{M}<N) ; I, \Omega$ : unit matrix and diagonalized eigenvalue matrix.

Power spectra of the generalized responses can be obtained thanks to the second-order Fourier transform of (3.5) with application of the SPT (2.4):

$$
\begin{aligned}
S_{\xi}(n)= & \left(\frac{1}{2} \rho U B\right)^{2}\left[H(n) \Phi C_{u}^{2} \Psi_{u}(n) \Lambda_{u}(n) \Psi_{u}^{* T} \mathrm{~K}(n)^{2} \Phi^{T} H(n)^{* T}\right. \\
& \left.+H(n) \Phi C_{w}^{2} \Psi_{w}(n) \Lambda_{w}(n) \Psi_{w}^{* T} \mathrm{~K}(n)^{2} \Phi^{T} H(n)^{* T}\right] \\
S_{\xi}(n)= & \left(\frac{1}{2} \rho U B\right)^{2}\left[H(n) A_{u}(n) \Lambda_{u}(n) K(n)^{2} A_{u}^{* T}(n) H^{T}(n)\right. \\
& \left.+H(n) A_{w}(n) \Lambda_{w}(n) K(n)^{2} A_{w}^{* T}(n) H(n)^{T}\right] \\
& A_{u}(n)=\sum_{j=1}^{\hat{M}} A_{u i j}(n)=\sum_{j=1}^{\hat{M}} \phi_{i}^{T} C_{u} \psi_{u j}(n) \\
& A_{w}(n)=\sum_{j=1}^{\hat{M}} A_{w i j}(n)=\sum_{j=1}^{\hat{M}} \phi_{i}^{T} C_{w} \psi_{w j}(n)
\end{aligned}
$$


where $A_{u}(n), A_{w}(n)$ : cross modal coefficient (CMC) matrices in which their elements $A_{u i j}(n), A_{w i j}(n)$ imply for influence of $j^{\text {th }}$ turbulent mode on $i^{\text {th }}$ structural one; $H(n)$ : frequency response function (FRF) matrix $H(n)=\operatorname{diag}\left(\left|H_{1}(n)\right|,\left|H_{2}(n)\right|, \ldots\left|H_{\bar{M}}(n)\right|\right)$ in which term of $\left|H_{i}(n)\right|$ denotes to FRF at natural frequency $n_{i},\left|H_{i}(n)\right|^{2}=\left[\left(1-\frac{n^{2}}{n_{1}^{2}}\right)^{2}+4 \zeta_{i}^{2} \frac{n^{2}}{n_{i}^{2}}\right]^{-1} ; K(n)^{2}$ : squared aerodynamic admittance function.

Spectra and root mean square (RMS) of the global responses are obtained:

$$
\begin{aligned}
S_{U}(n) & =\Phi S_{\xi}(n) \Phi^{T} ; \\
\sigma_{U}^{2} & =\int_{0}^{\infty} S_{U}(n) d n
\end{aligned}
$$

where $S_{U}(n), \sigma_{U}^{2}$ : spectra and root mean square of global responses, respectively.

Finally, global responses with respect to vertical, longitudinal and rotational directions can be combined from single-modal responses due to the principle of the squared root of the sum of the squares (SRSS):

$$
\sigma_{r}(n)=\sqrt{\sum_{i=1}^{\bar{M}_{r}} \sigma_{r, i}^{2}} ; r=h, p, a
$$

where $r$ denotes to displacement components: vertical $(h)$, longitudinal $(p)$, rotational $(a)$; $\bar{M}_{r}$ : number of component modes in response combination;

\section{NUMERICAL EXAMPLE AND DISCUSSION}

A cable-stayed bridge was taken for numerical example and investigation using the above-mentioned computational procedures. Bridge was spanned by $40.5+97+40.5=178$ m. Three-dimensional frame model was built thanks to the finite element method (FEM). Total 30 nodes were on bridge deck, while nodes 8,23 at pylons. First ten structural modes were analyzed (see Fig. 3). Damping ratios of each structural mode were assumed to be 0.005 . Mean wind velocity was taken at $20 \mathrm{~m} / \mathrm{s}$. It also assumed that the turbulent-induced forces act on deck only, those on towers and cables are negligible as usual. Aerodynamic static coefficients of cross section at balanced angle $\left(\alpha_{0}=0^{0}\right)$ and their first derivatives were experimentally determined as follows: $C_{L}=0.158, C_{D}=0.041, C_{M}=0.174, C_{L}^{\prime}=$ $3.73, C_{D}^{\prime} \approx 0, C_{M}^{\prime}=2.06$ (Le, 2004). One-sided auto spectral density functions of $u(t)$, $w(t)$ were obtained due to the Kaimail's and the Panofsky's models [15]:

$$
S_{u u}(n)=\frac{200 f u_{*}^{2}}{n(1+50 f)^{5 / 3}} ; \quad S_{w w}(n)=\frac{3.36 f u_{*}^{2}}{n\left(1+10 f^{5 / 3}\right)},
$$

where $f$ : non-dimensional coordinates $(f=n z / U) ; z$ : deck elevation $(z=10 \mathrm{~m}) ; u_{*}$ : friction velocity; $k, z_{0}$ : scale factor, roughness length $\left(k=0.4, z_{0}=0.025 \mathrm{~m}\right)$. Coherence function follows the equation (3.5) with decay factors: $c_{u}=10, c_{w}=6.5$ [18]. Squared aerodynamic admittance functions used the Liepmann's function:

$$
|\chi(n)|^{2}=\frac{1}{1+2 \pi^{2} n B / U} .
$$



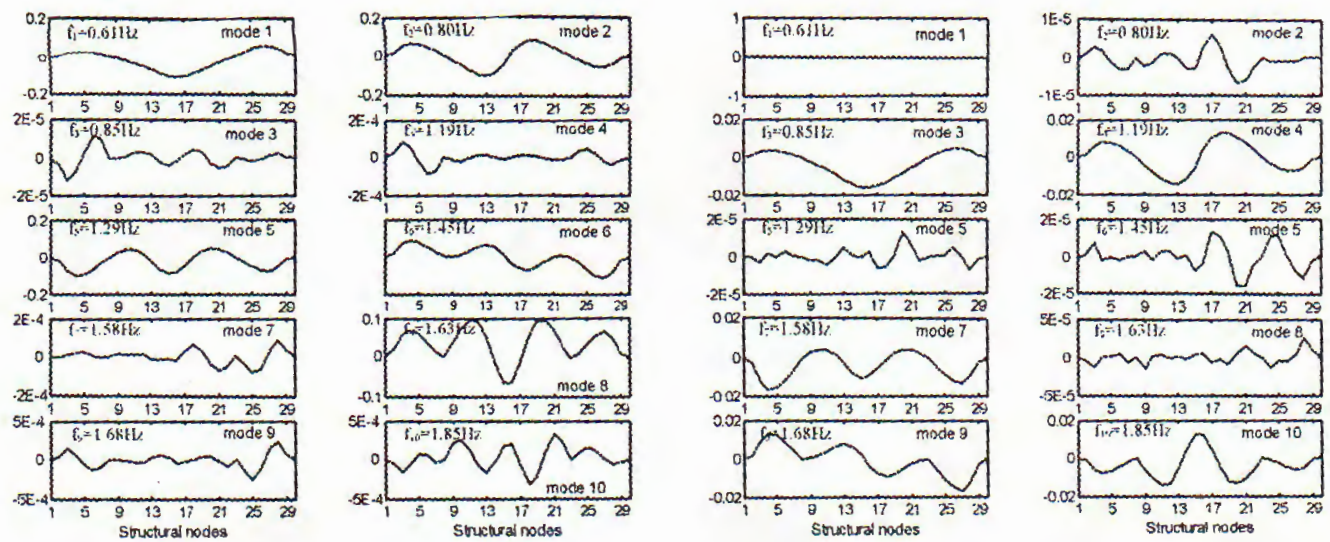

Fig. 3. Normalized structural modes: vertical (left) and rotational (right)
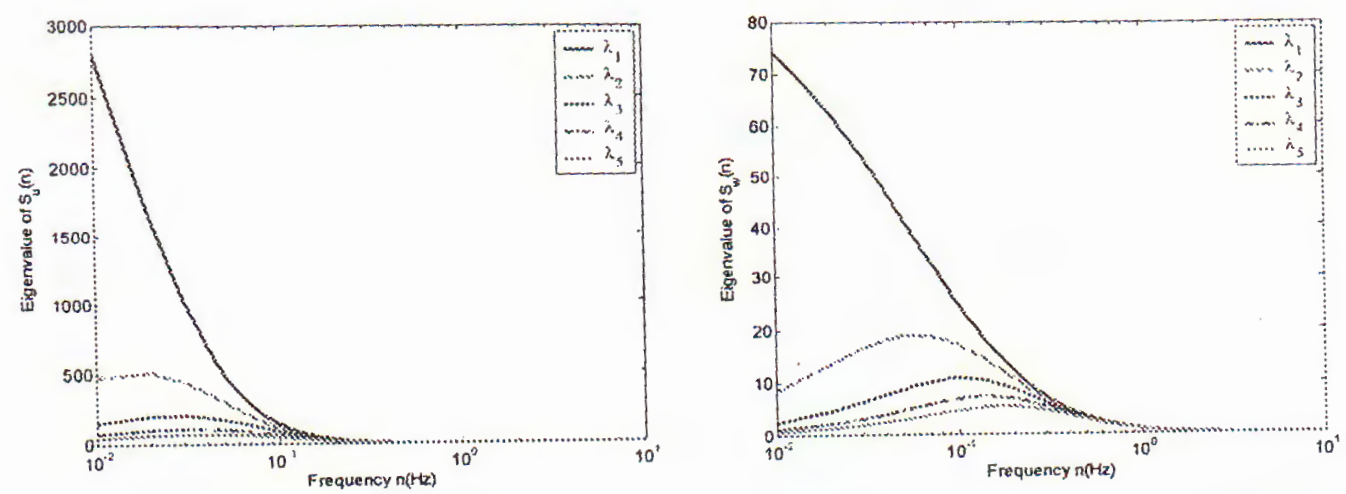

Fig. 4. First five spectral eigenvalues of spectral matrices $S_{u}\left(n^{\prime}(\right.$ left $)$ and $S_{w}(n)$ (right)

Cross spectral matrices $S_{u}(n), S_{w}(n)$ of spatially-correlated turbulent field $u(t), w(t)$ (acting on 30 deck nodes) were constructed. Spectral-based POD analysis was carried out to find entire 30 pairs of spectral eigenvectors and associated eigenvalues. Because the eigenvectors are orthogonal each other at each frequency, thus called as turbulent modes. Fig. 4 shows the first five spectral eigenvalues $\lambda_{1}(n) \div \lambda_{5}(n)$ on frequency band $0.01 \div 10$ Hz. It is observed that the first spectral eigenvalue $\lambda_{1}(n)$ exhibits much higher than the others on the very low frequency band $0.01 \div 0.2 \mathrm{~Hz}$ with the case of $u$-component, $0.01 \div 0.5 \mathrm{~Hz}$ with that of $w$-component, however, all spectral eigenvalues seem not to differ beyond these frequency thresholds. This can imply that only first pair of eigenvalue and eigenvector seems to be enough for representing and simulating the whole turbulent field at the very. low frequency bands, however, many more pairs are required at higher frequency bands.

The first three spectral eigenvectors $\psi_{v 1}(n), \psi_{v 2}(n), \psi_{v 3}(n) ; v=u, w$ (the turbulent modes) on the same spectral band $0 \div 10 \mathrm{~Hz}$ is expressed in Fig. 5 . It can be seen that the turbulent modes of $u-, w$ - components look like as symmetrically and asymmetrically sinusoidal waves, in which number of wave halves increases incrementally with the order 


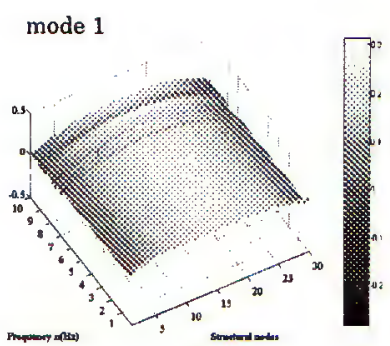

mode 1

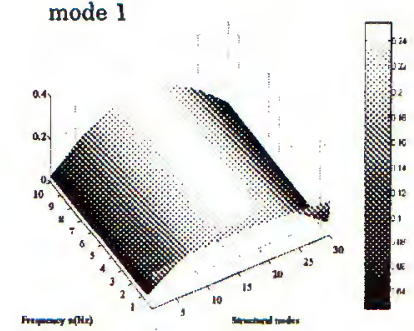

mode 2

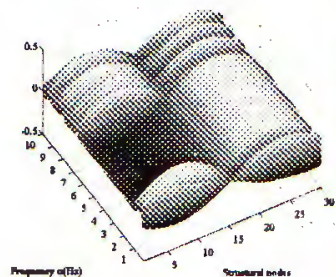

u- turbulence

mode 2

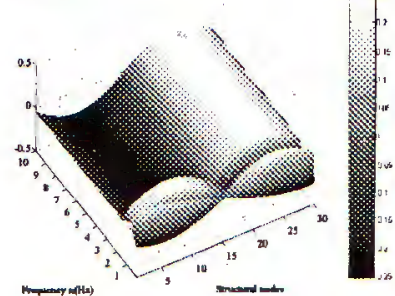

w- turbulence

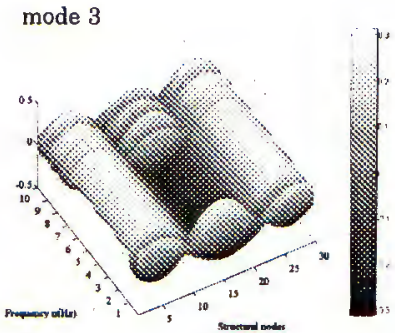

mode 3

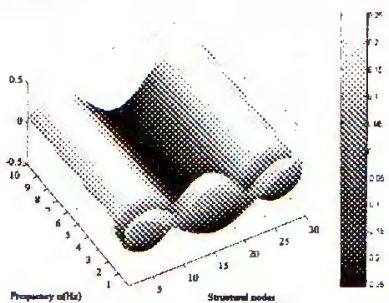

Fig. 5. First three spectral eigenvectors of $S_{u}(n)$ (upper) and of $S_{w}(n)$ (lower)

of eigenvectors. Shapes of the spectral eigenvectors of $u-, w$ - turbulent components, however, are unchanged during structurally natural frequency band $(0.61 \div 1.85 \mathrm{~Hz})$.
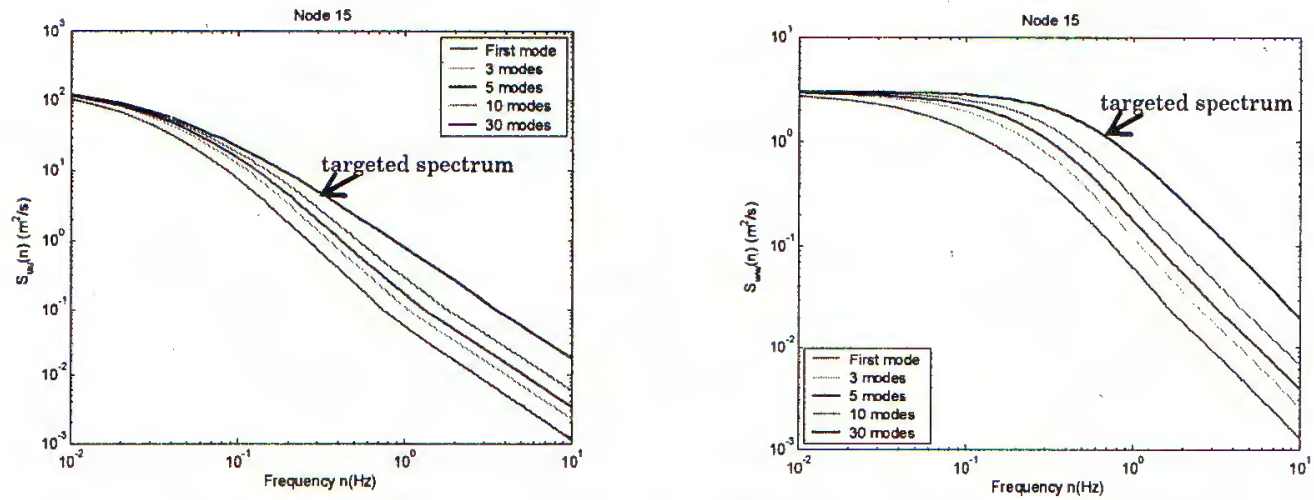

Fig. 6. Effect of number of turbulent modes on auto power spectra

Fig. 6 shows reconstruction of auto power spectra of $u, w$-turbulences at mid-span node 15 using limited number of truncated turbulent modes (due to Equation 2.4 with $\hat{M}=1 ; 3 ; 5 ; 10 ; 30$, here totally 30 turbulent modes imply for targeted value). Auto spectral values using truncated turbulent modes differ with increase of frequency band. At very low frequencies, only first or few turbulent modes are sufficient to reconstruct the auto spectral densities of turbulent field, however, many turbulent modes should be used at high frequencies. This finding is similar to that comment from Fig. 4. 
Effect of truncated turbulent modes on spectra of generalized responses (vertical and rotational displacements) at mid-span node 15 of some fundamentally structural modes at is expressed in Fig. 7.
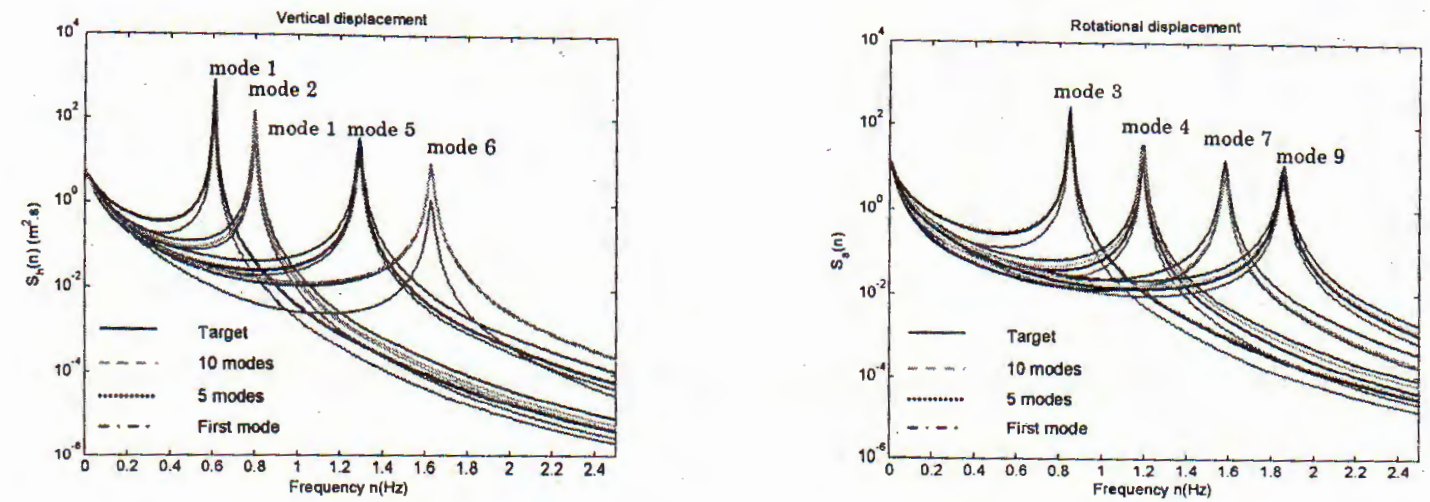

Fig. $\gamma$. Effect of number of turbulent modes on PSD of generalized responses
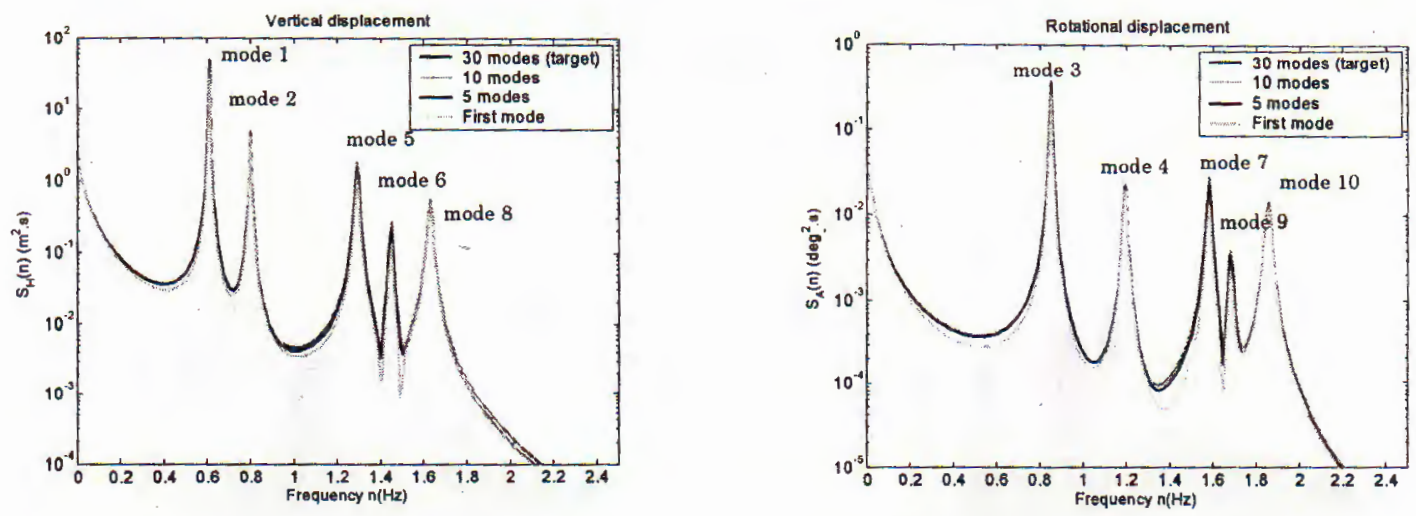

Fig. 8. Effect of number of turbulent modes on PSD of global responses at node 15

Fig. 8 shows the spectra of global responses at mid-span node 15 and effect of number of truncated turbulent modes. As can be seen from Figure 8 that no much different among cases of truncated turbulent modes, it is noted that first turbulent mode significantly contributes on spectra of responses. Spectral peaks or resonant responses, moreover, occur at structurally natural frequencies.

Fig. 9 expresses the root mean squares (RMS) of global responses at all deck nodes with emphasis on effect of truncated turbulent modes. Important role of the first turbulent mode on the global response is also observed. For example, the first turbulent mode, first 5 modes, first 10 modes contribute $29 \mathrm{~cm}(88 \%), 32 \mathrm{~cm}(97 \%), 32.6 \mathrm{~cm}(99 \%)$ on $32.7 \mathrm{~cm}$-maximum vertical displacement (totally 30 turbulent modes) and $0.032^{0}(91 \%)$, $0.033^{\circ}(97 \%), 0.034^{0}(100 \%)$ on $0.034^{\circ}$-maximum rotational displacement, respectively (see Fig. 9). 

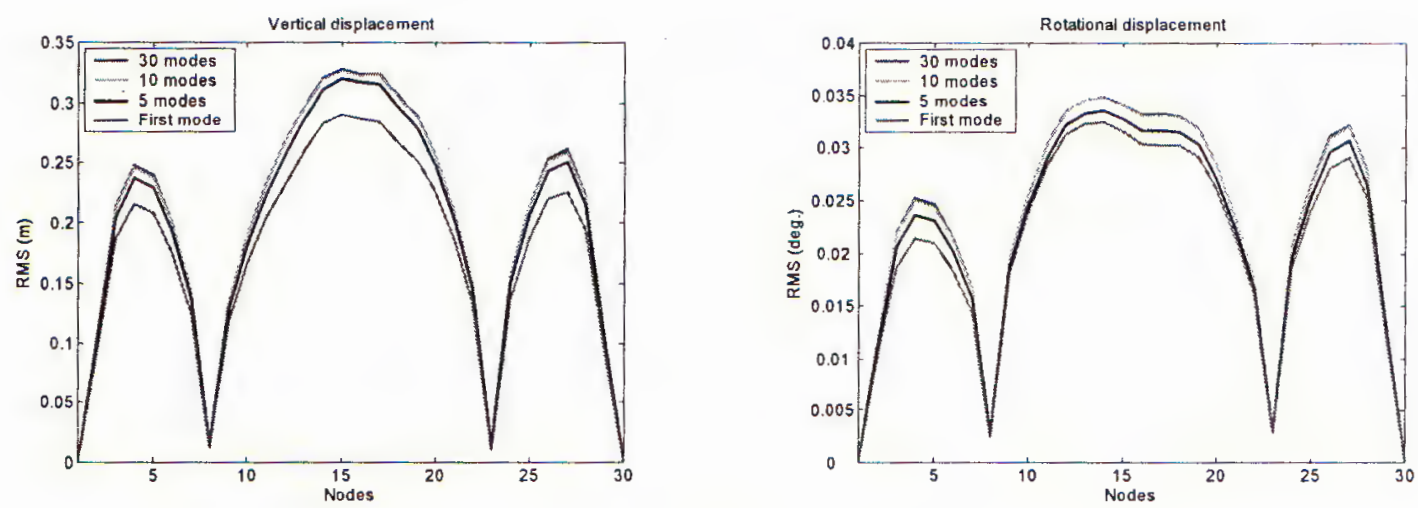

Fig. 9. Effect of number of turbulent modes on RMS of global responses at deck nodes

Influence of turbulent modes on structural ones has been investigated by cross modal coefficients defined in Equation 4.4b. Fig. 10 shows the cross modal coefficients between first 15 turbulent modes of $u$-, $w$-turbulent components and first 10 structural modes for lift or vertical displacement and moment or rotational displacement. It is found out that few turbulent modes (in this case, only first 9 turbulent modes) can excite structural modes, and higher turbulent modes are orthogonal to the structural modes.
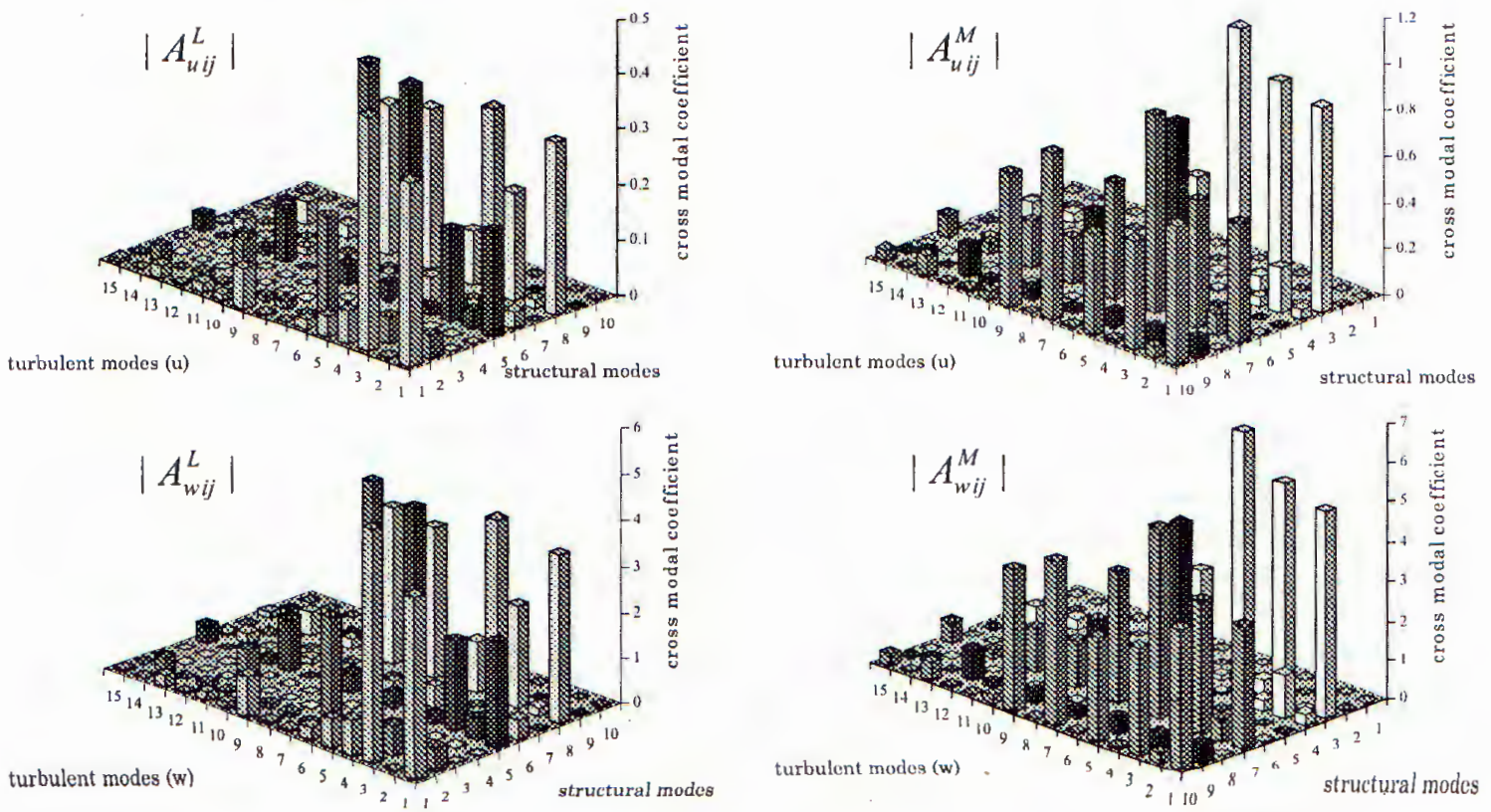

Fig. 10. Cross modal coefficients between turbulent modes and structural modes 
For more detail, first heaving mode ( $1^{\text {st }}$ structural mode) and first torsional mode ( $3^{\text {rd }}$ structural modes) are excited by the first and the third turbulent modes. Thus, gust response can be obtained by only accounting effective cross modal coefficients.

\section{CONCLUSION}

New and comprehensive approach on the gust response prediction of structures in the frequency domain using the proper orthogonal decomposition-based spectral proper transformation has been presented here. Spatially-correlated turbulent field has been represented due to orthogonally turbulent modes in which only limited number of lowest turbulent modes dominantly contributes on structural gust response. Further developments including digital simulation of multi-variate turbulent field using the spectral proper transformation as well as time-domain gust response prediction using the covariance-matrixbased POD and covariance proper transformation are going to be discussed in next paper.

Acknowledgments. First author would acknowledge Prof. Masaru Matsumoto, Prof. Hiromichi Shirato and Dr. Tomomi Yagi of the Bridge and Wind Engineering Laboratory, Department of Civil and Earth Resources Engineering, Kyoto University, Japan for their continuous supports during his fulfillment of this work.

\section{REFERENCES}

1. B. Bienkiewicz, Y. Tamura, H. J. Hann, H. Ueda, K. Hibi, Proper orthogonal decomposition and reconstruction of multi-channel roof pressure, Journal of Wind Engineering and Industrial Aerodynamics 54-55 (1995) 369-381.

2. G. Berkooz, P. Holmes, J. L. Lumley, The proper orthogonal decomposition in the analysis of turbulent flows, Annual Reviews on Fluid Mechanics 25 (1993), 539-575.

3. L. Carassale, G. Piccardo, G. Solari, Double modal transformation and wind engineering applications, ASCE Journal of Engineering Mechanics 127 (5) (1999) 432-439.

4. X. Chen, M. Matsumoto, A. Kareem, Time domain flutter and buffeting response analysis of bridges, ACSE Journal of Engineering Mechanics 126 (1) (2000) 7-16.

5. X. Chen, A. Kareem, Proper orthogonal decomposition-based modeling, analysis, and simulation of dynamic wind load effects on structures, ASCE Journal of Engineering Mechanics 131 (4) (2005) 325-339.

6. A. G. Davenport, Buffeting of a suspension bridge by storm winds. ASCE Journal of Structural Division (1962).

7. E. T. De Grenet, F. Ricciardelli, Spectral proper transformation of wind pressure fluctuations: application to a square cylinder and a bridge deck, Journal of Wind Engineering and Industrial Aerodynamics 92 (2004) 1281-1297.

8. T. H. Le, Experimental determination of aerodynamic coefficients of bridge sections in wind tunnel tests (in Vietnamese), J. Roads $\mathscr{E}$ Bridges 10 (2004).

9. T. H. Le, D. A. Nguyen, Multi-variate turbulent process decoupling and gust response prediction using spectral proper transformation, Proceedings of the $8^{\text {th }}$ National Conference on Solid Mechanics, Thai Nguyen, Viet Nam 25-26/8 (2006a).

10. T. H. Le, D. A. Nguyen, Digital simulation of turbulent field and gust response analysis of linelike structures based on proper orthogonal decomposition, Proceedings of the Int'l Conference on Nonlinear Analysis and Engineering Mechanics Today (NA-EMT2006), Ho Chi Minh City (2006b).

11. M. Matsumoto, H. Shirato, T. H. Le, Time and frequency domain gust response of bridges using proper orthogonal decomposition, Accepted for Int'l Conference on Wind Engineering (ICWE12), Caine Australia 7/2007. 
12. Y. S. Li, A. Kareem, Stochastic decomposition and application to probabilistic dynamics, ASCE Journal of Engineering Mechanics 121 (1) (1995) 162-174.

13. Y.C. Liang, P. Lee, W. Z. Lim, K. Lee, C. G. Xu, Proper orthogonal decomposition and its applications - part I: theory", Journal of Sound and Vibrations 252 (3) (2002) 527-544.

14. J. L. Lumley, Stochastic Tools in Turbulence, (1970) Academic Press.

15. E. Simiu, S. H. Scanlan, Wind Effects on Structures, John Wiley\&Sons, 1976.

16. G. Solari, L. Carassale, Modal transformation tools in structural dynamics and wind engineering, Wind Es Structures 3 - 4 (2000) 221-241.

17. Y. Tamura, S. Suganuma, H. Kikuchi, K. Hibi, Proper orthogonal decomposition of random wind pressure field, Journal of Fluid and Structures 13 (1999) 1069-1095.

18. G. Solari, F. Tubino, Gust response of long span bridges by double modal transformation, Proceedings of the $4^{\text {th }}$ European and African Conference on Wind Engineering (EACWE4) 2005.

Received June 25, 2007

\section{PHÉP CHUYẼ̃N PHỔ RIÊNG VÀ ÁP DỤNG TRONG XÅC ĐINH ĐÁP ỨNG NGẪU NHIÊN KHÍ ĐộNG LỰC CƯA CÔNG TRìnH}

Tải trọng gió ngẫu nhiên tác dụng lên kết cấu công trình nằm trong dòng khí rối thông thường được biểu diễn dưới dạng các quá trình ngẫu nhiên Gaussian đa chiều và đa biến. Tuy nhiên, việc xác tính toán ứng ngẫu nhiên do gió của kết cấu công trinh thường gặp khó khăn xuất phát từ việc chiếu các tải trọng gió ngẫu nhiên này lên các toạ độ suy rộng của kết cấu xác định tì̛ phân tích dạng thông thường. Trong trường hợp này thường áp dụng kỹ thuật tách nhằm tách trường tải trọng gió ngẫu nhiên thành các thành phần lực độc lập trọng toạ độ suy rộng của kểt cấu. Sau đó xác định đáp ứng của kết cấu trong các tọa độ suy rộng do kích động của các thành phần lực suy rộng này. Báo cáo này sẽ trình bày việc áp dụng phép tách trực giao riêng trên cơ sở phép chuyển phổ riêng trong miền tần số nhằm tách các quá trình lực gió ngẫu nhiên đa chiều tữ trường gió tương quan không gian thành các dạng tải trọng ngẫu nhiên trực giao, theo đó xác lập một cách tiếp cận mới trong tính toán đáp ứng ngẫu nhiên khí động lực của kết cấu công trình. Tính toán và khảo sát ví dụ cho kết cấu cầu dây văng. 